\title{
CHARACTERISTIC LENGTHS OF MAGNETIC FIELD IN MAGNETOHYDRODYNAMIC TURBULENCE
}

\author{
Jungyeon Cho and Dongsu Ryu ${ }^{1}$ \\ Department of Astronomy and Space Science, Chungnam National University, Daejeon, Korea; cho@canopus.cnu.ac.kr, ryu@canopus.cnu.ac.kr \\ Received 2009 April 9; accepted 2009 October 1; published 2009 October 19
}

\begin{abstract}
In the framework of turbulence dynamo, flow motions amplify a weak seed magnetic field through the stretching of field lines. Although the amplification process has been a topic of active research, less attention has been paid to the length scales of magnetic field. In this Letter, we describe a numerical study on characteristic lengths of magnetic field in magnetohydrodynamic turbulence. We considered the case of very weak or zero mean magnetic field, which is applicable to the turbulence in the intergalactic space. Our findings are as follows. (1) At saturation, the peak of magnetic field spectrum occurs at $\sim L_{0} / 2$, where $L_{0}$ is the energy injection scale, while the most energy containing scale is $\sim L_{0} / 5$. The peak scale of spectrum of projected, two-dimensional field is $\sim L_{0}$. (2) During the stage of magnetic field amplification, the energy equipartition scale shows a power law increase of $\sim t^{1.5}$, while the integral and curvature scales show a linear increase. The equipartition, integral, and curvature scales saturate at $\sim L_{0}, \sim 0.3 L_{0}$, and $\sim 0.15 L_{0}$, respectively. (3) The coherence length of magnetic field defined in the Faraday rotation measure (RM) due to the intergalactic magnetic field (IGMF) is related to the integral scale. We present a formula that expresses the standard deviation of $\mathrm{RM}, \sigma_{\mathrm{RM}}$, in terms of the integral scale and rms strength of the IGMF, and estimate that $\sigma_{\mathrm{RM}}$ would be $\sim 100$ and $\sim \mathrm{a}$ few $\mathrm{rad} \mathrm{m}^{-2}$ for clusters and filaments, respectively.
\end{abstract}

Key words: intergalactic medium - magnetic fields - MHD - turbulence

\section{INTRODUCTION}

It is well established that the universe is permeated with magnetic fields (see, e.g., Kronberg 1994), and yet their origin is not well understood (see Kulsrud \& Zweibel 2008, and references therein). The problem of cosmic magnetism can be divided into two parts- the origin of seed fields and their amplification. In this Letter, we are concerned with the latter.

If a weak seed magnetic field is introduced into a turbulent medium, flow motions stretch field lines and amplify the field. Such turbulent amplification has been studied since 1950s (see, e.g., Batchelor 1950; Kulsrud \& Anderson 1992; Kulsrud et al. 1997; Cho \& Vishniac 2000; Brandenburg \& Subramanian 2005; Schekochihin \& Cowley 2007; Ryu et al. 2008; Cho et al. 2009). The amplification goes through three stages. (1) Eddy motions in turbulence are fastest on the smallest scale, which is the dissipation scale. Therefore, the stretching of magnetic field lines occurs most actively at the dissipation scale first, and the magnetic energy grows exponentially. (2) The exponential growth stage ends when the magnetic energy becomes comparable to the kinetic energy at the dissipation scale. The follow-up stage is characterized by a linear growth of magnetic energy, and a gradual shift of the peak of magnetic field spectrum to larger scales. (3) The amplification of magnetic field stops when the total magnetic energy becomes comparable to the kinetic energy. A final, statistically steady, saturation stage is reached.

Ryu et al. (2008) proposed a scenario in which turbulent flow motions are induced via the cascade of the vorticity generated at cosmological shocks during the formation of the largescale structure. Based on a model of turbulent amplification, they estimated the strength of the intergalactic magnetic field (IGMF): $\langle B\rangle \sim$ a few $\mu \mathrm{G}$ in clusters, $\sim 0.1 \mu \mathrm{G}$ around clusters, and $\sim 10 \mathrm{nG}$ in filaments. In their model, the intracluster medium

\footnotetext{
1 Author to whom any correspondence should be addressed.
}

is at a stage close to the saturation one. But the intergalactic medium (IGM) in filaments are still at the linear growth stage.

The IGMF has been observed with the Faraday rotation measure (RM); for instance, Clarke et al. (2001) in clusters and $\mathrm{Xu}$ et al. (2006) outside clusters. For the IGMF with $\langle\boldsymbol{B}\rangle=0$, the mean value of RM is expected to vanish, and the standard deviation represents the observation (see, e.g., Ryu et al. 1998). Hence,

$$
\sigma_{\mathrm{RM}}=0.81 \bar{n}_{e} B_{\| \mathrm{rms}} l \sqrt{\frac{L}{l}} \mathrm{rad} \mathrm{m}^{-2},
$$

where $n_{e}, B_{\|}$, and $l$ are in units of $\mathrm{cm}^{-3}, \mu \mathrm{G}$, and pc, respectively, has been used to extract the strength of the IGMF. Here, $B_{\| \text {rms }}$ is the rms strength of the line-of-sight magnetic field, $l$ is the coherence length, and $L$ is the path length. But the formula has been applied without a clear definition of the coherence length.

Here, we study length scales of magnetic field in magnetohydrodynamic (MHD) turbulence with very weak or zero mean magnetic field, intended for application to the IGMF. Several characteristic lengths, which have been introduced in previous works, are considered. Characteristic lengths of initial seed fields in the early universe could be either very large or very small, depending on how the seed fields were generated. However, the field structure at the linear growth and saturation stages is independent of the seed fields as long as they are sufficiently weak (see, e.g., Cho et al. 2009). Therefore, the characteristic lengths should be determined by the energy injection scale (or the outer scale) only. And based on a scaling argument, Schekochihin \& Cowley (2007) stated that in the linear growth stage the scale at which the stretching is most active grows as $l_{s} \propto t^{3 / 2}$.

In this Letter, through numerical simulations, we investigate the growth and saturation of characteristic lengths. Then, we show how the coherence length in the RM due to the IGMF is defined in terms of the characteristic lengths, and present a formula for $\sigma_{\mathrm{RM}}$. And based on the model of Ryu et al. (2008), we estimate $\sigma_{\mathrm{RM}}$ for clusters and filaments. 


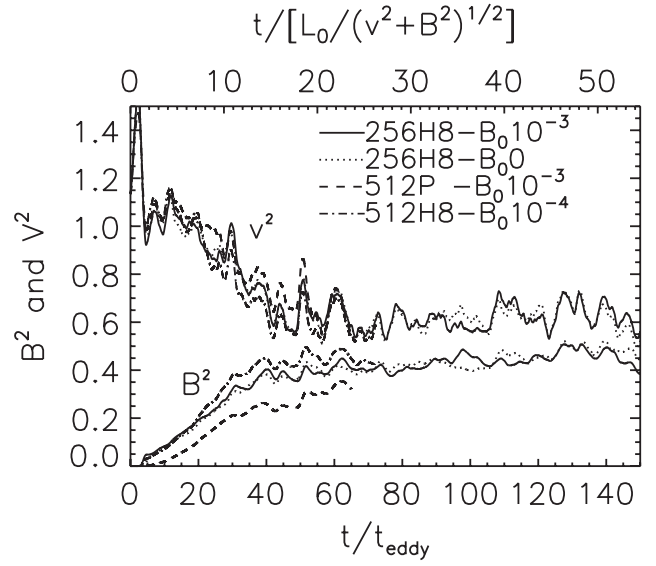

Figure 1. Time evolution of $V^{2}$ and $B^{2}$. Time is given both in units of the eddy turnover time defined with the vorticity around the energy injection scale at saturation, $t_{\text {eddy }} \equiv 1 / \omega_{\text {injection, }}$ (bottom) as in Ryu et al. (2008) and in units of the energy injection scale divided by $\sqrt{V^{2}+B^{2}}$ at saturation (top) as in Cho et al. (2009).

\section{SIMULATIONS}

We solved the incompressible MHD equations in a periodic box of size $2 \pi$ using a pseudospectral code. We drove turbulence in Fourier space. The forcing function has the following form: $\boldsymbol{f}(\boldsymbol{x}, t)=\sum_{j=1}^{22} \boldsymbol{f}\left(\boldsymbol{k}_{j}\right) \exp \left(i \boldsymbol{k}_{j} \cdot \boldsymbol{x}\right)+$ complex conjugate, where $\boldsymbol{f}\left(\boldsymbol{k}_{j}\right)$ is a complex vector that is perpendicular to the wavevector $\boldsymbol{k}_{j}$. The 22 forcing components are nearly isotropically distributed in the range $2 \leqslant k \leqslant \sqrt{12}$, where $k=|k|$. The phase of each forcing component randomly fluctuates, but it has a correlation time of approximately unity. The amplitude of each forcing component also randomly fluctuates. On average, each forcing component injects similar amount of energy. ${ }^{2}$ In physical space this forcing $\boldsymbol{f}(\boldsymbol{x}, t)$ corresponds to statistically homogeneous driving on large scales. Only the solenoidal component of the velocity field was driven. ${ }^{3}$ The strength of energy injection was tuned, so without magnetic field the rms velocity becomes unity, $V_{\mathrm{rms}} \sim 1$, at saturation. In this representation, $\boldsymbol{V}$ can be viewed as the velocity measured in units of the rms velocity of the system. The density is unity and the magnetic field is multiplied by $\sqrt{4 \pi}$ in our simulations, so $\boldsymbol{B}$ can be viewed as the Alfvén speed in the same units. The magnetic field consists of the uniform background field and the fluctuating field: $\boldsymbol{B}=\boldsymbol{B}_{0}+\boldsymbol{b}$. At $t=0$, the magnetic field had either weak uniform component (when $B_{0} \neq 0$ ) or only random components (when $B_{0}=0$; Run $256 H 8-B_{00}$, see below), and the velocity had a support between $2 \leqslant k \leqslant 4$ in the wavevector space. We considered only the case where the kinetic viscosity, $v$, is equal to the magnetic diffusivity, $\eta$. See Cho \& Vishniac (2000) and Cho et al. (2009) for further details of simulations.

Simulations are denoted with $X Y-B_{0} Z$, where $X=256$ or 512 refers to the number of grid points in each spatial direction, $Y=H 8$ or $P$ refers to hyper (and their order) or

\footnotetext{
2 The wavenumbers used are 2 (three components), $\sqrt{6}$ (12 components), 3 (three components), and $\sqrt{12}$ (four components). Therefore, the peak of energy injection occurs at $k_{0} \approx 2.5$, and the energy injection scale is $L_{0} \sim 2.5$. A similar discrete sampling of forcing can be found in Tao et al. (1993), where they used 13 components.

3 Since we deal with incompressible turbulence, we applied solenoidal forcing. However, in general cases, the properties of turbulence may depend on the nature of forcing (see, e.g., Federrath et al. 2008, for compressible turbulence).
}

physical viscosity/diffusivity, and $Z$ refers to the strength of mean magnetic field. In this Letter, Runs $256 \mathrm{H} 8-B_{00}, 256 H 8$ $B_{0} 10^{-3}, 512 P-B_{0} 10^{-3}$, and $512 H 8-B_{0} 10^{-4}$ are discussed. In $256 H 8-B_{00}$, the mean field was zero, and initially the spectrum of magnetic field peaked at $k \sim 70$. All the runs have either very weak or zero mean magnetic field, since we intend to apply the results to turbulence in the IGM, ${ }^{4}$ as noted in the Introduction. Besides the initial magnetic field configuration, runs have either different numerical resolution or different viscosity/diffusivity to explore their effects. In $512 P-B_{0} 10^{-3}$ physical viscosity/ diffusivity was used, while in others hyperviscosity/diffusion was used to extend the inertial range. ${ }^{5}$ Runs with $256^{3}$ grid points are a subset of the simulations used in Cho et al. (2009). For the properties of turbulence with different initial magnetic fields, please refer an extensive study in Cho et al. (2009). Figure 1 shows the time evolution of $V^{2}$ and $B^{2}$ in four simulations. Here, the kinetic energy and magnetic energy densities are $V^{2} / 2$ and $B^{2} / 2$. Although the four simulations have different setups, their time evolution looks similar. We can clearly see three stages of magnetic energy evolution. In this limit of week or zero mean field, the resulting turbulence is globally almost isotropic.

\section{VARIOUS LENGTH SCALES OF MAGNETIC FIELD}

Peak scale of spectrum of magnetic field, $L_{E(k)}$ : Figure 2 (left) shows the spectra of velocity and magnetic field at an epoch of saturation. In $E_{v}(k)$, the peak occurs at the energy injection scale. Note that the peak appears at $k=2$ rather that at 2.5, which is a numerical artifact of discrete binning of $k$. In $E_{b}(k)$, the peak occurs at a smaller scale.

Largest energy containing scale of magnetic field, $L_{k E(k)}$ : with $E(k) \propto 1 / k$ representing a power spectrum that contains equal amount of energy in each decade of $k$, the peak scale of $k E(k)$ defines the largest energy containing scale. Figure 2 (left) shows that for the magnetic field the peak of $k E_{b}(k)$ occurs at a scale even smaller than that of $E_{b}(k)$.

Peak scale of spectrum of projected magnetic field, $L_{E(k) / k}$ : when a three-dimensional (3D) scalar quantity is projected onto a two-dimensional (2D) plane, the energy spectrum of the projected quantity becomes $E^{2 D}(k) \propto E^{3 D}(k) / k$ (see, e.g., Cho \& Lazarian 2002). It is a bit more complicated for vector quantities, but the qualitative behavior should be similar. Figure 2 (left) shows that for the magnetic field the peak of $E_{b}(k) / k$ occurs at a scale close to that of $E_{v}(k)$. Note that the largest energy containing scale of projected magnetic field corresponds to the peak scale of $k E_{b}^{2 D}(k)$, which is the same as the peak scale of $E_{b}(k)$.

The time evolution and saturation of $L_{E(k)}, L_{k E(k)}$, and $L_{E(k) / k}$, normalized with the energy injection scale $L_{0}$, are shown in Figure 2 (right). The rugged profiles are again a consequence of discrete binning of $k$. Two points are noteworthy. (1) At

\footnotetext{
4 The origin of seed magnetic fields in the IGM can be either cosmological or astrophysical. Theories in favor of cosmological origin suggest that weak seed magnetic fields were created in the early universe or during the structure formation era. Our initial magnetic field intends to mimic the cosmological origin of seed fields.

5 It is well known that hyperdissipation causes the bottleneck effect, unphysical flattening of energy spectrum near the dissipation range. However, since the bottleneck effect is negligible at small wavenumbers, we believe the effect does not alter the shape of the magnetic energy spectrum at small wavenumbers. Most length scales discussed in this Letter rely on the shape of magnetic energy spectrum at small wavenumbers. Therefore, we believe the use of hyperdissipation does not affect our results much. Indeed, our results show that the length scales do not strongly depend on numerical resolution or forms of dissipation.
} 

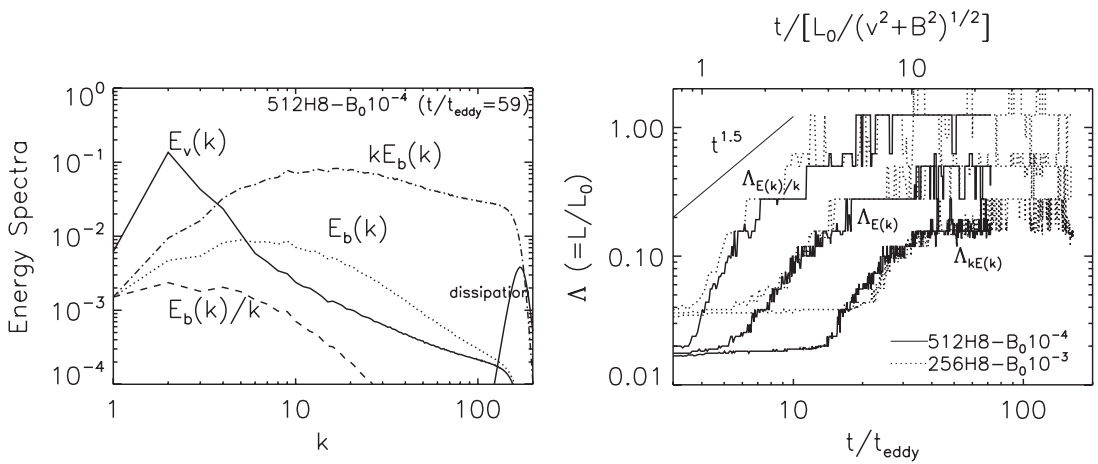

Figure 2. Left panel: spectra of velocity, $E_{v}(k)$, and magnetic field, $E_{b}(k)$, at $t / t_{\text {eddy }}=59$. The dissipation spectrum is also shown. Although hyperdissipation affects the spectra near the dissipation scale, it does not strongly influence the shapes of the spectra at small wavenumbers. Right panel: time evolution of peak scales of $E_{b}(k), k E_{b}(k)$, and $E_{b}(k) / k$. The $\Lambda$ 's are the scales normalized with $L_{0}$.

saturation, $L_{E(k)} \sim L_{0} / 2, L_{k E(k)} \sim L_{0} / 5$, and $L_{E(k) / k} \sim L_{0}$. (2) The growth pattern of those scales in the linear growth stage is not clear owing to the ruggedness in the profiles, but it seems to be between $\sim t$ and $\sim t^{1.5}$.

Energy equipartition scale, $L_{\mathrm{eq}}$ : the energy equipartition wavenumber, $k_{\text {eq }}$, is defined by

$$
\int_{k_{\mathrm{eq}}}^{k_{\max }} E_{v}(k) d k=\int_{0}^{k_{\max }} E_{b}(k) d k
$$

The time evolution and saturation of $L_{\mathrm{eq}}$ are shown in Figure 3 (left). We expect that the stretching of magnetic field lines is most active at this scale. If then, $L_{\text {eq }}$ should represent $l_{s}$ (see the Introduction) and follow $\sim t^{1.5}$ in the linear growth stage (Schekochihin \& Cowley 2007). Indeed, $L_{\text {eq }}$ grows with a power-law index consistent with the theoretical prediction in Figure 3 (left). At saturation, $L_{\mathrm{eq}}$ becomes close to $L_{0}$.

Integral scale, $L_{\mathrm{int}}$ : the length scale of magnetic field (and velocity too) is often characterized with the integral scale, which is defined by

$$
L_{\text {int }}=2 \pi \frac{\int E_{b}(k) / k d k}{\int E_{b}(k) d k} .
$$

It is well known that the integral scale has the same order of magnitude as the longitudinal and transversal integral scales, $L_{l}$ and $L_{t}$, respectively, and in incompressible isotropic turbulence (with reflection invariance on average) they are related by $L_{l}=2 L_{t}=(3 / 8) L_{\text {int }}$ (see Monin \& Yaglom 1975).

Curvature scale, $L_{\mathrm{curv}}$ : we also consider a typical radius of curvature of field lines, $L_{\text {curv }}$. We define it as the distance $r$ at which the average correlation drops to $1 / e$,

$$
\frac{\langle\boldsymbol{B}(\boldsymbol{x}) \cdot \boldsymbol{B}(\boldsymbol{x}+\boldsymbol{r})\rangle_{x}}{\langle\boldsymbol{B}(\boldsymbol{x}) \cdot \boldsymbol{B}(\boldsymbol{x})\rangle_{x}}=\frac{1}{e},
$$

where the two points at separation $r$ are located along the same magnetic field line and the average is taken over $\boldsymbol{x}$. The factor $1 / e$ is an arbitrary choice.

The time evolution and saturation of $L_{\text {int }}$ and $L_{\text {curv }}$ are shown in Figure 3 (left). We calculated $L_{\text {curv }}$ only for runs with $256^{3}$ grid points. Unlike $L_{\text {eq }}, L_{\text {int }}$ and $L_{\text {curv }}$ seem to grow linearly in the linear growth stage (although the reason of the linear growth is not clear). At saturation, $L_{\text {int }} \sim 0.3 L_{0}$ and $L_{\text {curv }} \sim 0.15 L_{0}$. Hence, for instance, we model the growth and saturation of $L_{\mathrm{int}}$ as

$$
L_{\text {int }} \sim \begin{cases}(0.3 / 45) L_{0} \times t / t_{\text {eddy }}, & \text { if } t / t_{\text {eddy }}<45 \\ 0.3 L_{0}, & \text { if } t / t_{\text {eddy }} \geqslant 45\end{cases}
$$

where the eddy turnover time is defined with the vorticity around the energy injection scale at saturation as $t_{\text {eddy }} \equiv 1 / \omega_{\text {injection }}$.

\section{FARADAY ROTATION MEASURE}

With $\langle\mathrm{RM}\rangle=0$ for the IGMF, the standard deviation of RM

$$
\sigma_{\mathrm{RM}}=0.81 \bar{n}_{e}\left\langle\left(\int_{0}^{L} B_{\|} d s\right)^{2}\right\rangle^{1 / 2} \operatorname{rad~m^{-2}} .
$$

Note that in this work the density is assumed to be constant. Here, $\int B_{\|} d s$ is the projected, 2D magnetic field.

Without loss of generality, we take $x$ as the line-of-sight direction. The projected field can be written as ${ }^{6}$

$$
\begin{aligned}
\int_{0}^{2 \pi} B_{\|} d s & \equiv \int_{0}^{2 \pi} B_{x} d x \approx \int_{0}^{2 \pi} d x \sum_{k_{x}, k_{y}, k_{z}=-N / 2}^{N / 2} \tilde{B}_{x}(\boldsymbol{k}) e^{i \boldsymbol{x} \cdot \boldsymbol{k}} \\
& =2 \pi \sum_{k_{y}, k_{z}=-N / 2}^{N / 2} \tilde{B}_{x}\left(0, k_{y}, k_{z}\right) e^{i\left(y k_{y}+z k_{z}\right)}
\end{aligned}
$$

where we used $\int_{0}^{2 \pi} d x e^{i x k_{x}}=2 \pi \delta_{0, k_{x}}$. Then, the square-average of the projected field becomes

$$
\begin{aligned}
\left\langle\left(\int_{0}^{2 \pi} B_{\|} d s\right)^{2}\right\rangle & =\frac{1}{(2 \pi)^{2}} \int_{0}^{2 \pi} d y \int_{0}^{2 \pi} d z\left(\int_{0}^{2 \pi} B_{x} d x\right)^{2} \\
& =(2 \pi)^{2} \sum_{k_{y}, k_{z}=-N / 2}^{N / 2}\left|\tilde{B}_{x}\left(k_{x}=0, k_{y}, k_{z}\right)\right|^{2} \\
& \approx(2 \pi)^{2} \int d k_{y} d k_{z}\left|\tilde{B}_{x}\left(k_{x}=0, k_{y}, k_{z}\right)\right|^{2} \\
& =\frac{(2 \pi)^{2}}{2} \int \frac{E_{b}(k)}{k} d k
\end{aligned}
$$

In the last step, $\left\langle\left|\tilde{B}_{x}\right|^{2}\right\rangle_{k_{x}=0}=(1 / 2)\left\langle|\tilde{B}|^{2}\right\rangle_{k_{x}=0}$ statistically in the $k_{x}=0$ plane and $E_{b}(k)=4 \pi k^{2}\left(|\tilde{B}|^{2} / 2\right)$ were used, and

\footnotetext{
6 We considered turbulence in a cubic box of size $2 \pi$, as noted in Section 2 . That is, we assumed a flow of period $2 \pi$ in the three directions of space (see Lesieur 2008, for further details). In this case, we have a usual Fourier series expansion: $B_{x}(\boldsymbol{x}) \approx \sum_{k_{x}, k_{y}, k_{z}=-N / 2}^{N / 2} \tilde{B}_{x}(\boldsymbol{k}) e^{i \boldsymbol{x} \cdot \boldsymbol{k}}$ and $\tilde{B}_{x}(\boldsymbol{k})=\frac{1}{(2 \pi)^{3}} \int_{0}^{2 \pi} d x$ $\int_{0}^{2 \pi} d y \int_{0}^{2 \pi} d z B_{x}(\boldsymbol{x}) e^{-i \boldsymbol{x} \cdot \boldsymbol{k}} \approx \frac{1}{N^{3}} \sum_{l, m, n=0}^{N-1} \int B_{x}\left(\boldsymbol{x}_{l m n}\right) e^{-i \boldsymbol{x}_{l m n} \cdot \boldsymbol{k}}$, where $N$ is the number of grid points in each side. Here, $\boldsymbol{k}=\left(k_{x}, k_{y}, k_{z}\right)$, and $\boldsymbol{x}_{l m n}$ denotes the coordinate of a grid point $(l, m, n)$ in the computational grid.
} 

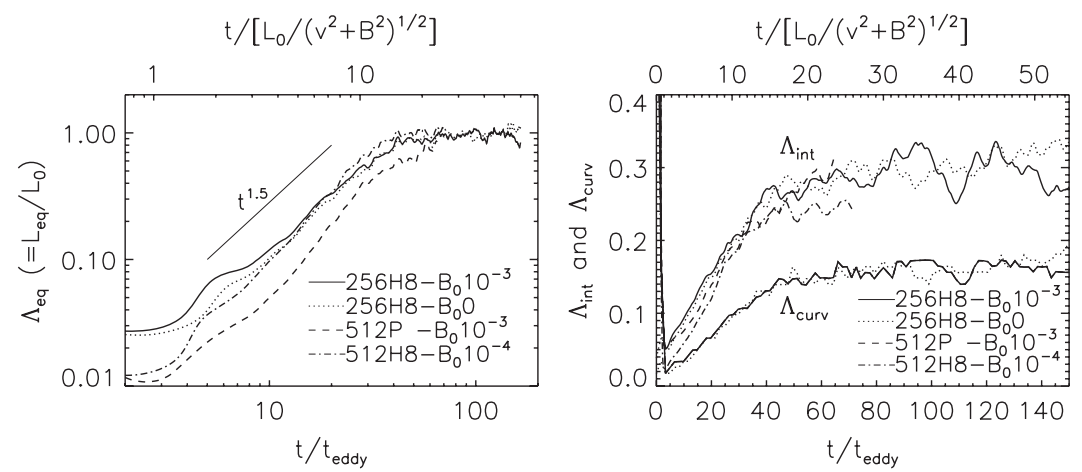

Figure 3. Time evolution of the energy equipartition scale (left panel) and the integral and curvature scales (right panel). The $\Lambda$ 's are the scales normalized with $L_{0}$.

$d k_{y} d k_{z}$ was substituted with $2 \pi k d k$. Finally, using $\left\langle B^{2}\right\rangle / 2=$ $\int E_{b}(k) d k$

$$
\begin{aligned}
\left\langle\left(\int_{0}^{2 \pi} B_{\|} d s\right)^{2}\right\rangle & =\frac{\left\langle B^{2}\right\rangle(2 \pi)^{2}}{4} \frac{\int E_{b}(k) / k d k}{\int E_{b}(k) d k} \\
& =\frac{\left\langle B^{2}\right\rangle L_{\mathrm{int}}(2 \pi)}{4} .
\end{aligned}
$$

So far, we have assumed that the box size is $2 \pi$. When the box size is $L$ (or, when the path length is $L$ ), ${ }^{7} 2 \pi$ 's in Equation (9) should be replaced by $L$. Hence, the standard deviation of RM becomes

$$
\sigma_{\mathrm{RM}}=0.81 \bar{n}_{e} \frac{B_{\mathrm{rms}} \sqrt{L_{\mathrm{int}} L}}{2} \mathrm{rad} \mathrm{m}^{-2}
$$

Since $B_{\| \mathrm{rms}}=B_{\mathrm{rms}} / \sqrt{3}$, the coherence length used in Equation (1) should be given as $l=(3 / 4) L_{\text {int }}$.

Equation (10) can be applied to estimate RMs due to the IGMF. Here, we employ the model of Ryu et al. (2008) for turbulence and magnetic field in the IGM. In clusters, turbulence is near the saturation stage with $t / t_{\text {eddy }} \sim 30$, where $t \equiv t_{\text {age }}$, the age of the universe, and $t_{\text {eddy }} \equiv \omega_{\mathrm{rms}}^{-1}$. From Equation (5), $L_{\text {int }} / L_{0} \sim 0.2$. If we take the energy injection scale $L_{0} \sim 100 \mathrm{kpc}$, which is approximately the scale height of cluster core, $L_{\text {int }} \sim 20 \mathrm{kpc}$. With $\bar{n}_{e} \sim 10^{-3} \mathrm{~cm}^{-3}$, $B_{\mathrm{rms}} \sim$ a few $\mu \mathrm{G}$, and the path length $L \sim 1 \mathrm{Mpc}$, we get $\sigma_{\mathrm{RM}} \sim 100 \mathrm{rad} \mathrm{m}^{-2}$ for clusters, which agrees with the observed RMs in clusters (Clarke et al. 2001). In filaments, on the other hand, with $t / t_{\text {eddy }} \sim 10$, turbulence is expected to be still in the linear growth stage, and $L_{\text {int }} / L_{0} \sim 1 / 15$. We may take the energy injection scale $L_{0} \sim 5 \mathrm{Mpc}$, which is the typical thickness of filaments. It is also the typical radius of curvature of cosmological shocks in filaments (Ryu et al. 2003), which would be the major sources to drive turbulence there. The power spectrum of $\boldsymbol{v}_{\text {curl }}$, the curl component of flow motions which satisfies the relation $\nabla \times \boldsymbol{v}_{\text {curl }} \equiv \nabla \times \boldsymbol{v}$, in the large-scale structure of the universe peaks around $5 \mathrm{Mpc}$ too (Ryu \& Kang 2008). Then, $L_{\text {int }} \sim 300 \mathrm{kpc}$. The magnetic field strength in filaments quoted in Ryu et al. (2008) is $\langle B\rangle \sim 10 \mathrm{nG}$. But we note that the value depends on how it is averaged. With the data of Ryu et al. (2008), $\left\langle B^{2}\right\rangle^{1 / 2} \sim$ a few $\times 10 \mathrm{nG}$, $\langle\rho B\rangle /\langle\rho\rangle \sim 0.1 \mu \mathrm{G}$, and $\left\langle(\rho B)^{2}\right\rangle^{1 / 2} /\left\langle\rho^{2}\right\rangle^{1 / 2} \sim$ a few $\times 0.1 \mu \mathrm{G}$, in the warm-hot intergalactic medium (WHIM) with $T=10^{5}$ $10^{7} \mathrm{~K}$ which mostly composes filaments. The average value of $\left\langle(\rho B)^{2}\right\rangle^{1 / 2} /\left\langle\rho^{2}\right\rangle^{1 / 2}$ should be most relevant to RM. Then, $\sigma_{\mathrm{RM}}$

\footnotetext{
7 The periodic simulation box size can be used as a proxy for the physical path length of the system (see, e.g., discussion in Lesieur 2008).
}

for filaments, if they intersect the line of sight with right angle, would be

$$
\begin{aligned}
\sigma_{\mathrm{RM}} \sim & 1.5\left(\frac{\bar{n}_{e}}{10^{-5} \mathrm{~cm}^{-3}}\right)\left(\frac{B_{\mathrm{rms}}}{0.3 \mu \mathrm{G}}\right) \\
& \times\left(\frac{L_{\mathrm{int}}}{300 \mathrm{kpc}} \frac{L}{5 \mathrm{Mpc}}\right)^{1 / 2} \mathrm{rad} \mathrm{m}^{-2} .
\end{aligned}
$$

Normally filaments would not intersect the line of sight with right angle. Smaller angles result in larger path lengths and so larger $\sigma_{\mathrm{RM}}$, and then the typical value of $\sigma_{\mathrm{RM}}$ for filaments could be a few $\mathrm{rad} \mathrm{m}^{-2}$. We note that the values of $|\mathrm{RM}|$ toward the Hercules and Perseus-Pisces superclusters reported in Xu et al. (2006) are an order of magnitude larger than the above value, and Xu et al. (2006) quoted the path length, $L$, which is about 2 orders of magnitude larger.

\section{SUMMARY}

Our findings are summarized. (1) We studied different characteristic scales of magnetic field in MHD turbulence with very weak or zero mean magnetic field. They saturate at $\sim 0.1-1 L_{0}$, where $L_{0}$ is the energy injection scale. (2) During the linear growth stage of magnetic energy, the energy equipartition scale follows $L_{\mathrm{eq}} \propto t^{3 / 2}$, while the integral scale follows $L_{\text {int }} \propto t$. (3) The integral scale (actually (3/4) $L_{\text {int }}$ ) is the relevant scale for RM. We obtained a new formula for the standard deviation of RM, $\sigma_{\mathrm{RM}}$ (see Equation (10)). (4) We estimated $\sigma_{\mathrm{RM}}$ for filaments as well as for clusters of galaxies.

Finally, we note that our findings are based on a small number of incompressible numerical simulations. They appear to be rather insensitive to numerical resolution and forms of dissipation. However, when different initial conditions or different types of forcing are used, some of the results may be different, a question which should be investigated in the future.

We thank the anonymous referee for useful suggestions. J.C. was supported in part by the Korea Research Foundation (KRF2006-331-C00136). D.R. was supported in part by the Korea Science and Engineering Foundation (R01-2007-000-20196-0). J.C. and D.R. were also supported by the Korea Foundation for International Cooperation of Science and Technology (K20702020016-07E0200-01610).

\section{REFERENCES}

Batchelor, G. 1950, Proc. R. Soc. Lond. A, 201, 405

Brandenburg, A., \& Subramanian, K. 2005, Phys. Rep., 417, 1

Cho, J., \& Lazarian, A. 2002, ApJ, 575, L63

Cho, J., \& Vishniac, E. 2000, ApJ, 538, 217 
Cho, J., Vishniac, E., Beresnyak, A., Lazarian, A., \& Ryu, D. 2009, ApJ, 693, 1449

Clarke, T. E., Kronberg, P. P., \& Böhringer, H. 2001, ApJ, 547, L111

Federrath, C., Klessen, R. S., \& Schmidt, W. 2008, ApJ, 688, L79

Kulsrud, R., \& Anderson, S. 1992, ApJ, 396, 606

Kulsrud, R., Cen, R., Ostriker, J., \& Ryu, D. 1997, ApJ, 480, 481

Kulsrud, R., \& Zweibel, E. 2008, Rep. Prog. Phys., 71, 046901

Kronberg, P. P. 1994, Rep. Prog. Phys., 57, 325

Lesieur, M. 2008, Turbulence in Fluids (4th ed.; New York: Springer)

Monin, A. S., \& Yaglom, A. M. 1975, Statistical Fluid Mechanics (Cambridge: MIT Press)
Ryu, D., \& Kang, H. 2008, in ASP Conf. Ser. 385, Numerical Modelings of Space Plasma Flows: Astronum 2007, ed. N. V. Pogorelov et al. (San Francisco, CA: ASP), 44

Ryu, D., Kang, H., \& Biermann, P. L. 1998, A\&A, 335, 19

Ryu, D., Kang, H., Cho, J., \& Das, S. 2008, Science, 320, 909

Ryu, D., Kang, H., Hallman, E., \& Jones, T. W. 2003, ApJ, 593, 599

Schekochihin, A., \& Cowley, S. 2007, in Magnetohydrodynamics: Historical Evolution and Trends, ed. S. Molokov et al. (Berlin: Springer), 85

Tao, L., Cattaneo, F., \& Vainshtein, S. I. 1993, NATO Advanced Study Institute: Solar and Planetary Dynamos, 303

Xu, Y., Kronberg, P. P., Habib, S., \& Dufton, Q. W. 2006, ApJ, 637, 19 